TIF-UNIMI-2020-1

\title{
On the Sudakov form factor, and a factor of two
}

\author{
Stefano Forte \\ Tif Lab, Dipartimento di Fisica, Università di Milano and \\ INFN, Sezione di Milano, \\ Via Celoria 16, I-20133 Milano, Italy
}

\begin{abstract}
I answer a question that Roman Jackiw asked me, and I draw some lessons from the answer. The question is: why is the Sudakov form factor larger by a factor of two, if computed for off-shell fermions, in comparison to the onshell case? The answer sheds some light on the interplay between infrared and collinear singularities - and the importance of factors of two.

Contribution to the volume Roman Jackiw - 80th Birthday Festschrift
\end{abstract}

\section{Master of scientific style}

Much can be said about working under Roman's supervision in the mideighties: it was an absorbing, intense, at times exhilarating, at times stressful experience. A formidable array of ideas to take in, concepts to grasp, and good practices to learn, often delivered as a side remark, accompanied by a grin! Ranging from the way to write displayed equations in a paper ("it is called an equation because it has an equal sign"), to the importance of choosing the symbols when performing a calculation ("one should not pick letters at random from the alphabet"). Some perks, too, such as going for dinner at the Harvard faculty club with Steven Hawking and Sidney Coleman — including the task of steadying the former's wheelchair in the minivan that took us there.

Overall, it amounted to a lesson of scientific method, and of scientific style: delivered mostly by example. One thing I understood - the painful way, as I am prone to algebraic mistakes - is the importance of details when

\footnotetext{
${ }^{1}$ See the contribution by Michiel Bos in this volume.
} 
performing a computation. Roman used to retell the story of someone who published a perturbative computation in which he had guessed the value of a high-order term without actually calculating it — only to be belied by the explicit result once Roman got round to determine it.

Not so long ago, I came to think about it again. It was the Summer of 2017, I was spending some time at the Aspen center for physics, and Roman, coincidentally also there, took me out for lunch. The conversation at some point revolved on some then-recent work of mine [1] on QCD resummation. Roman mentioned that he had worked on related topics around the time of his PhD thesis [2]: he had computed perturbatively the high-momentum transfer limit of the QED vertex function, which is double logarithmic, and correctly guessed the exponentiation of the double logs. As our lunch was going on, Roman then abruptly asked me whether I knew that the coefficient of the double $\log$ is by a factor of two larger off-shell in comparison to the on shell-result, and whether I knew a simple physical reason for that.

I didn't know.

Continuing the discussion with Roman via email, after I got back home, I realized that I couldn't immediately come up with an answer. I also subsequently realized that this point is typically not discussed, or even mentioned in textbooks. In fact, what is commonly known as "the Sudakov form factor" is the on-shell result, yet the original[3] Sudakov calculation applies to the off-shell case, and the factor two difference usually goes unnoticed. Indeed, as Roman pointed out to me, in a recent paper from the Russian school 4 it is incorrectly stated ${ }^{2}$ that the same result applies in the on-shell and offshell cases, only with a different choice of infrared regulator. I asked various experts on QCD, where the Sudakov exponentiation plays an important role, and none was aware of this.

Answering Roman's question is the purpose of this note.

\section{The vertex function and the Sudakov form factor}

The computation performed by Roman 2] determines to all orders the highenergy behavior of the vertex function in Quantum Electrodynamics (QED) (see Fig. 1). The genesis of this paper has been recounted by Roman[5]: his advisor, Ken Wilson, suggested him as a thesis project to derive this highenergy behavior, which had been previously obtained by Sudakov 3 in the off-shell case, using renormalization-group (RG) methods: both as a way of

\footnotetext{
${ }^{2}$ See in particular the discussion after Eq. (34) of Ioffe's paper. 4 ]
} 
validating the then-novel $R G$ techniques, and also, of obtaining the on-shell result.

The way to attack and solve this problem using RG techniques was only found several years later [6, 7] (see also Sect. 4.3 below), but Roman did manage to tackle it by direct computation using an eikonal approximation, which was known to Wilson, and systematically developed by Weinberg 8 into what is now known as light-cone field theory $\left.\right|^{3}$

The result found by Roman 2 is that in the high-energy limit the vertex function is equal to

$$
\Gamma^{\mu}\left(p_{1}, p_{2}\right)=\gamma^{\mu} \Gamma\left(p_{1}^{2}, p_{2}^{2}, q^{2}\right) ;
$$

where to one loop (Fig. 1)

$$
\Gamma^{(1), \text { off }}\left(p_{1}^{2}, p_{2}^{2}, q^{2}\right)=-\frac{\alpha}{2 \pi} \ln \left|\frac{q^{2}}{p_{1}^{2}}\right| \ln \left|\frac{q^{2}}{p_{2}^{2}}\right|
$$

for off-shell fermions with virtualities $p_{i}^{2}$, while for on-shell fermions

$$
\Gamma^{(1), \text { on }}\left(m^{2}, m^{2}, q^{2}\right)=-\frac{\alpha}{4 \pi} \ln ^{2} \frac{|q|^{2}}{\mu^{2}}
$$

where $\mu$ is an infrared regulator (i.e. a photon mass).

To all perturbative orders the one-loop result exponentiates:

$$
\begin{aligned}
\Gamma^{\mathrm{off}}\left(p_{1}^{2}, p_{2}^{2}, q^{2}\right) & =\exp -\frac{\alpha}{2 \pi} \ln \left|\frac{q^{2}}{p_{1}^{2}}\right| \ln \left|\frac{q^{2}}{p_{2}^{2}}\right|, \\
\Gamma^{\mathrm{on}}\left(m^{2}, m^{2}, q^{2}\right) & =\exp -\frac{\alpha}{4 \pi} \ln ^{2} \frac{|q|^{2}}{\mu^{2}} .
\end{aligned}
$$

All these results hold to double-logarithmic accuracy, i.e. up to terms with a lower power of $\ln |q|^{2}$.

The result Eq. (4) is in agreement with the previous result of Sudakov [3], which had been subsequently reproduced by others [11], who attempted to determine the on-shell result but did not obtain the correct answer and failed to prove exponentiation. The results Eqs. (4.5) are also given in the volume devoted to QED of "Landau's" theoretical physics course[12], first published in 1974 (after Landau's death): for the off-shell result Sudakov [3] is cited, while the on-shell result is written in the form

$$
\bar{\Gamma}^{(1), \text { on }}\left(m^{2}, m^{2}, q^{2}\right)=-\frac{\alpha}{4 \pi}\left(\ln ^{2}\left|\frac{q^{2}}{m^{2}}\right|+4 \ln \left|\frac{q^{2}}{m^{2}}\right| \ln \left|\frac{m}{\mu}\right|\right),
$$

${ }^{3}$ A textbook discussion of the eikonal approximation is e.g. given by Sterman [9, while a presentation of light-cone field theory can be found in recent summer school proceedings [10]. 


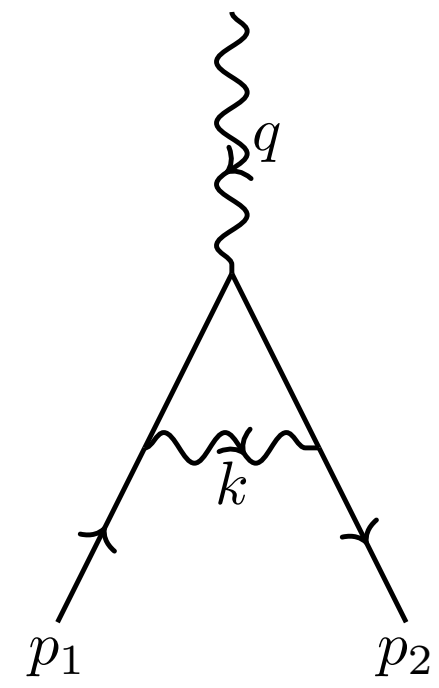

Figure 1: The one-loop vertex function.

which of course coincides with Eq. (3) up to terms which are not logarithmic in $q^{2}$ :

$$
\bar{\Gamma}^{(1), \text { on }}\left(m^{2}, m^{2}, q^{2}\right)=\Gamma^{(1), \text { on }}\left(m^{2}, m^{2}, q^{2}\right)+\frac{\alpha}{4 \pi} \ln ^{2} \frac{m^{2}}{\mu^{2}} .
$$

It is clear that, contrary to what one might naively think (and contrary to what sometimes stated explicitly[4]) the on-shell result is not simply obtained by setting $p_{1}^{2}=p_{2}^{2}=\mu^{2}$ in the off-shell one - rather, it is twice as large. Why?

Answering this question requires a computation of the vertex function, starting at one loop. Rather than the vertex function itself, however, it is more instructive to look at its real-emission counterpart. Indeed, it is the imaginary part of the photon propagator in the diagram of Fig. 1 which leads to the double-logarithmic behavior Eqs. (2,5) [2, 12. This imaginary part can be extracted using the standard cutting rule

$$
\operatorname{Disc} \frac{1}{k^{2}+i \epsilon}=-2 \pi i \delta\left(k^{2}\right) \Theta\left(k^{0}\right) \text {. }
$$

This transforms the vertex function into the interference of two real emission diagrams (see Fig. 2). Of course, it is is this one-to-one correspondence of virtual and real emission contributions which guarantees the cancellation of infrared singularities for sufficiently inclusive physical observables. ${ }^{4}$

\footnotetext{
${ }^{4}$ See Chapter 13 of Weinberg's treatise 13 , for a modern discussion.
} 


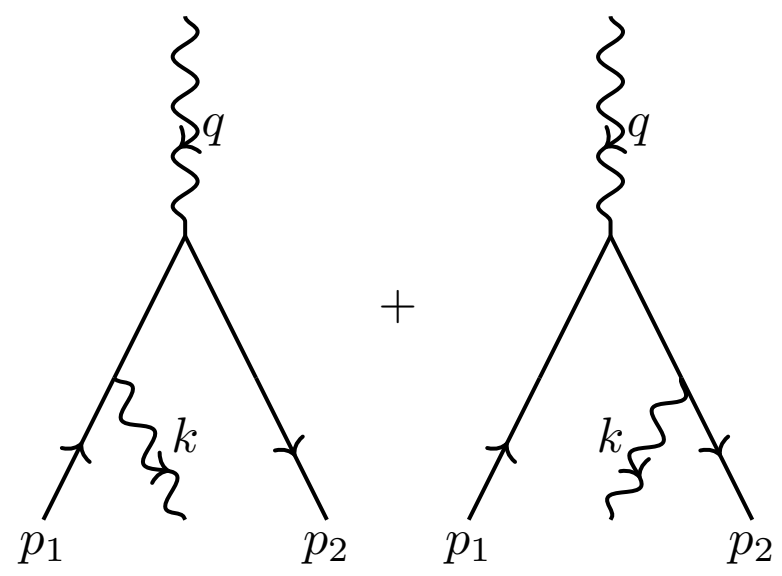

Figure 2: The real emission diagrams corresponding to the vertex function of Fig. 1

I will first, present in Sect. 3 a direct computation of these real-emission contributions, viewed as contributions to the decay amplitude of a virtual photon into a fermion-antifermion pair, in the rest frame of the virtual photon. This computation reproduces the result of Eqs. (2-3), and it gives a first hint on its origin. For a complete clarification, however, it is useful to look at the problem in a different frame: namely, by viewing the diagrams of Fig. 2 as contributions to Drell-Yan-like production of a virtual photon in a fermionantifermion collision, in the center-of-mass frame of the colliding fermions. In Sect. 4 I will show that the origin of the difference between on-shell and off-shell can be traced to a different interplay of soft and collinear singularities in either case. Exponentiation then ensues from the factorized structure of phase-space, which can be proved using an argument [1] developed in order to combine soft and collinear resummation.

\section{Computing the vertex function}

Consider the decay of an off-shell photon with momentum $q$. Of course, this means that the incoming fermion of Fig. 2 now is an outgoing antifermion, and the result in the kinematics of Fig. 2 can be recovered by crossing. However, because the double log behavior only depends on the modulus of the momentum transfer, the computation can be indifferently performed in either kinematics. For simplicity we consider the case of massless fermions,

\footnotetext{
${ }^{5}$ The computation presented in this section is based on unpublished notes by Paolo Nason [14].
} 
though it can be checked explicitly[14] that results are unchanged with a finite fermion mass.

Double logs Eqs. (2, 3) arise due to the soft region of integration over the momentum $k$ of the emitted photon. The amplitude for emission of a soft photon is obtained multiplying the amplitude for the process without soft photon by an eikonal factor ${ }^{6}$, so that in this limit the amplitude for the process of Fig. 2 is given by

$$
M=M_{0} e\left(\frac{2 p_{1}^{\mu}}{\left(p_{1}+k\right)^{2}}-\frac{2 p_{2}^{\mu}}{\left(p_{2}+k\right)^{2}}\right),
$$

where $M_{0}$ is the amplitude without the extra photon. The square amplitude is then

$$
|M|^{2}=-\left|M_{0}\right|^{2} e^{2} \frac{8 p_{1} \cdot p_{2}}{\left(p_{1}+k\right)^{2}\left(p_{2}+k\right)^{2}},
$$

and the desired real-emission amplitude is find integrating this over the phase space of the emitted photon:

$$
d \Phi_{k}=\frac{k^{2} d k d \cos \theta d \phi}{2 E(2 \pi)^{3}}=\frac{k d E d \cos \theta}{8 \pi^{2}},
$$

where $E$ and $k$ are respectively the energy and modulus of the three-momentum of the emitted photon, and in the last step we have used $\frac{d E}{k}=\frac{d k}{E}$, and integrated over the azimuth $\phi$.

The calculation is performed in the on-shell case by assuming a small photon mass $\mu$ as a regulator, so $\left(p_{i}+k\right)^{2}=2 p_{i} \cdot k+\mu^{2}$, and in the off-shell case by assuming $p_{i}^{2}>0$ so $\left(p_{i}+k\right)^{2}=2 p_{i} \cdot k+p_{i}^{2}$.

\subsection{On-shell fermions}

In the rest frame of the decaying photon the square amplitude is

$$
\begin{aligned}
|M|^{2} & =-\left|M_{0}\right|^{2} e^{2} \frac{4 s}{\left[E \sqrt{s}(1-\beta \cos \theta)+\mu^{2}\right]\left[E \sqrt{s}(1+\beta \cos \theta)+\mu^{2}\right]} \\
& =-\left|M_{0}\right|^{2} e^{2} \frac{4}{E^{2}\left[(1-\beta \cos \theta)+\frac{\mu^{2}}{E \sqrt{s}}\right]\left[(1+\beta \cos \theta)+\frac{\mu^{2}}{E \sqrt{s}}\right]},
\end{aligned}
$$

where $E=k^{0}$ is the emitted photon's energy, $\sqrt{s}=\sqrt{\left(p_{1}+p_{2}\right)^{2}}=2 p_{i}^{0}=$ $\sqrt{\left|q^{2}\right|}$ is the energy of the fermion-antifermion system, and

$$
\beta=\sqrt{1-\frac{\mu^{2}}{E^{2}}}=1-\frac{\mu^{2}}{2 E^{2}}\left(1+O\left(\mu^{2} / E^{2}\right)\right) .
$$

\footnotetext{
${ }^{6}$ See Chapter 13 of Weinberg's treatise 13 .
} 
Integrating over the emitted photon's phase space Eq. (11) we get

$$
\begin{aligned}
& \int d \Phi_{k}|M|^{2} \\
& =-\left|M_{0}\right|^{2} \frac{e^{2}}{2 \pi^{2}} \int \frac{E d E d \cos \theta}{E^{2}\left[\left(1-\cos \theta+\cos \theta \frac{\mu^{2}}{2 E^{2}}+\frac{\mu^{2}}{E \sqrt{s}}\right)\right]\left[\left(1+\cos \theta-\cos \theta \frac{\mu^{2}}{2 E^{2}}+\frac{\mu^{2}}{E \sqrt{s}}\right)\right]}
\end{aligned}
$$

This leads to logarithmic behavior either when $\theta \rightarrow 1$ or $\theta \rightarrow-1$, corresponding to the region in which the emitted photon is respectively collinear to $p_{1}$ or $p_{2}$. These two collinear and anti-collinear contributions are the same, and we get

$$
\int d \Phi_{k}|M|^{2}=-2\left|M_{0}\right|^{2} \frac{e^{2}}{4 \pi^{2}} \int \frac{d E d \cos \theta}{E(1-\cos \theta)}+\text { non log }
$$

where we have for definiteness written the collinear contribution, while introducing a factor of two in order to account for the anticollinear one, and we have retained the leading term in an expansion in $\cos \theta$ about $\cos \theta=1$, as well as in an expansion of $\mu^{2}$ about $m^{2}=0$, so the second square bracket in the denominator of Eq. (15) just reduces to a factor of two.

Performing the angular integral we immediately get

$$
\int d \Phi_{k}|M|^{2}=-2\left|M_{0}\right|^{2} \frac{\alpha}{\pi} \int \frac{1}{2} \frac{d E^{2}}{E^{2}} \ln \left[\left(\frac{\mu^{2}}{2 E^{2}}+\frac{\mu^{2}}{E \sqrt{s}}\right)^{-1}\right],
$$

where we have introduced the fine-structure constant $\alpha=\frac{e^{2}}{4 \pi}$. The double integral comes from the infrared region of integration over the energy $E$ of the emitted fermion, hence we can neglect the second term in the argument of the log, and we get, keeping only double logarithmic terms,

$$
\int d \Phi_{k}|M|^{2}=-\left|M_{0}\right|^{2} \frac{\alpha}{2 \pi} \ln ^{2} \frac{|q|^{2}}{\mu^{2}}
$$

where the upper limit of integration over energy is of course just $\sqrt{s} / 2$; indeed, the argument of the log is fixed by dimensional analysis.

The real emission contribution should be compared to the square of the virtual one, which leads to an extra factor of two in the real emission case, so this result exactly matches Roman's result [2] for the on-shell vertex function Eq. (3). 


\subsection{Off-shell fermions}

If the fermions are off-shell, it is now the fermion virtuality which regulates the collinear singularity, so that no massive photon regulator is needed. The square amplitude is then

$$
|M|^{2}=-\left|M_{0}\right|^{2} e^{2} \frac{4 s}{\left[E \sqrt{s}(1-\beta \cos \theta)+p_{1}^{2}\right]\left[E \sqrt{s}(1+\beta \cos \theta)+p_{2}^{2}\right]}\left[1+O\left(p_{i}^{2} / s\right)\right]
$$

where now the equalities $2 p_{i}^{0} \approx \sqrt{\left|q^{2}\right|} \approx \sqrt{s}$ all hold up to terms of order $p_{i}^{2} / s$, and

$$
\beta_{i}=\sqrt{1-\frac{p_{i}^{2}}{\left(p_{i}^{0}\right)^{2}}}=1-\frac{2 p_{i}^{2}}{s}\left[1+O\left(p_{i}^{2} / s\right)\right] .
$$

Integrating over the photon's phase space we have again a pair of collinear and anticollinear singularities:

$$
\begin{aligned}
& \int d \Phi_{k}|M|^{2} \\
& =-\left|M_{0}\right|^{2} \frac{e^{2}}{2 \pi^{2}} \int \frac{E d E d \cos \theta}{\left[E(1-\cos \theta)+\cos \theta E \frac{2 p_{1}^{2}}{s}+\frac{p_{1}^{2}}{\sqrt{s}}\right]\left[E(1+\cos \theta)-\cos \theta E \frac{2 p_{2}^{2}}{s}+\frac{p_{2}^{2}}{\sqrt{s}}\right]} .
\end{aligned}
$$

However, there are two differences: the form of the collinear cutoff, which now depends on the virtuality, $p_{i}^{2}$ rather than the photon mass $\mu^{2}$ and also, the form of the energy denominator - the second square bracket in the denominator of Eq. (21) — which is now also cut off. Indeed, focusing as before on the collinear contribution (with a factor of two accounting for the anti-collinear one) we get

$$
\int d \Phi_{k}|M|^{2}=-2\left|M_{0}\right|^{2} \frac{e^{2}}{4 \pi^{2}} \int \frac{E d E d \cos \theta}{E\left[(1-\cos \theta)+\frac{2 p_{1}^{2}}{s}+\frac{p_{1}^{2}}{E \sqrt{s}}\right]\left[E+\frac{2 p_{2}^{2}}{2 \sqrt{s}}\right]}+\text { non log, }
$$

where again we have kept the leading terms as $\cos \theta \rightarrow 1$.

Performing the angular integral we now get

$$
\int d \Phi_{k}|M|^{2}=-2\left|M_{0}\right|^{2} \frac{\alpha}{\pi} \int \frac{d E}{E+\frac{p_{2}^{2}}{\sqrt{s}}} \ln \left[\left(\frac{2 p_{1}^{2}}{s}+\frac{p_{1}^{2}}{E \sqrt{s}}\right)^{-1}\right]
$$


so it is apparent that the logarithmic integration over $E$ is cut off by $\frac{p_{2}^{2}}{\sqrt{s}}$. It is now the first term in the argument of the log which is subleading, and performing the integral over the energy gives

$$
\int d \Phi_{k}|M|^{2}=-\left|M_{0}\right|^{2} \frac{\alpha}{\pi} \ln \frac{s}{p_{1}^{2}} \ln \frac{s}{p_{2}^{2}}=-\left|M_{0}\right|^{2} \frac{\alpha}{\pi} \ln \frac{|q|^{2}}{p_{1}^{2}} \ln \frac{|q|^{2}}{p_{2}^{2}}
$$

up to single logarithmic terms. This is indeed twice as big as the on-shell result Eq. (18), and thus it exactly matches Roman's result[2].

\section{Infrared and collinear singularities}

Having reproduced the result of Eqs. (2, 3), and in particular the factor two difference between on- and off-shell, we would now like to understand the origin of this difference. Comparing Eqs. (15.22), it is clear that in both cases the double log stems from a collinear and an infrared singularity, respectively coming from the integral over the angle and the energy of the emitted photon. The difference resides in the way the singularities are regulated by the photon mass, or by the virtuality: however, the factor two appears somewhat haphazard, as it looks like the reason why Eqs. (3) is twice as large is that the integration variable is the energy $E$, rather than $E^{2}$, with the remaining $\sqrt{s}$ dependence contained in the cutoff.

However, a more transparent physical interpretation appears if we consider the same computation, but in a different frame. Namely, we view the amplitude of Fig. 2 as the production of an off-shell photon in the annihilation of a fermion-antifermion pair, in the center-of-mass reference frame of the incoming fermions. The physics is then similar to the familiar one of Drell-Yan production in QCD (in which the fermions are quarks).

\subsection{The Sudakov parametrization}

It is then convenient to introduce a Sudakov-like parametrization of the momentum $k$ of the emitted photon (which, in the QCD analogy, would be an emitted gluon):

$$
\begin{aligned}
k & =(1-x) \frac{p_{1}+p_{2}}{2}+y \frac{p_{1}-p_{2}}{2}+k_{\mathrm{T}}, \\
& =x_{1} p_{1}+x_{2} p_{2}+k_{\mathrm{T}}
\end{aligned}
$$


where $k_{\mathrm{T}} \cdot p_{1}=k_{\mathrm{T}} \cdot p_{2}=0$ is a space-like transverse momentum vector, such that $k_{T}^{2}=-\left|k_{T}\right|^{2}$, and of course

$$
\begin{aligned}
& x_{1}=\frac{1}{2}[(1-x)+y], \\
& x_{2}=\frac{1}{2}[(1-x)-y],
\end{aligned}
$$

so that either $\left(x_{1}, x_{2}\right)$ or $(x, y)$ can be used according to convenience.

In the center-of-mass frame of the incoming fermion-antifermion pair the energy of the emitted photon is

$$
E=(1-x) \frac{\sqrt{s}}{2}
$$

while its longitudinal momentum component

$$
k_{z} \equiv y \frac{p_{1}-p_{2}}{2}
$$

is entirely fixed by the on-shell condition

$$
\left|k_{z}\right|=\sqrt{E^{2}-\left|k_{\mathrm{T}}\right|^{2}}=y \frac{\sqrt{\left|p_{1}-p_{2}\right|^{2}}}{2}
$$

where in the general off-shell case $\left|p_{1}-p_{2}\right|^{2}=s-2\left(p_{1}^{2}+p_{2}^{2}\right)$. Of course in the off-shell case $k_{z}$ Eq. (31) is the longitudinal momentum only up to terms proportional to the difference of the two virtualities. Solving for $y$ we get

$$
y= \pm \sqrt{(1-x)^{2}-\frac{4\left|k_{\mathrm{T}}\right|^{2}}{s}}\left(1+O\left(p_{i}^{2} / s\right)\right) .
$$

The advantage of this choice of parametrization is seen by writing the phase-space of the emitted photon, which now takes the form

$$
d \Phi_{k}=\frac{\left|k_{\mathrm{T}}\right| d\left|k_{\mathrm{T}}\right| d \phi d k_{z}}{2 E(2 \pi)^{3}}=\frac{d\left|k_{\mathrm{T}}\right|^{2} d E}{4\left|k_{z}\right|\left(4 \pi^{2}\right)},
$$

instead of the previous Eq. (11). Using Eqs. 29.31) we get

$$
d \Phi_{k}=\frac{1}{4\left(4 \pi^{2}\right)} \frac{d x d\left|k_{\mathrm{T}}\right|^{2}}{\sqrt{(1-x)^{2}-\frac{4\left|k_{\mathrm{T}}\right|^{2}}{s}}}\left(1+O\left(p_{i}^{2} / s\right)\right) .
$$

This last form exposes the phase-space origin[7, 1] of the soft and collinear singularity: if the squared amplitude behaves as $|M|^{2} \underset{\left|k_{\mathrm{T}}\right| \rightarrow 0}{\sim} \frac{1}{\left|k_{\mathrm{T}}\right|}$, the $k_{\mathrm{T}}$ 
integration is logarithmic; but then as $\left|k_{\mathrm{T}}\right| \rightarrow 0$ the square root factor in the denominator reduces to $1-x$ and the $x$ integration also becomes logarithmic, in the $x \rightarrow 1$ limit in which the energy of the emitted photon Eq. (29) vanishes.

This can be exposed by rewriting, in the limit as $\left|k_{\mathrm{T}}\right|^{2} \rightarrow 0$

$$
\begin{aligned}
d \Phi_{k} & =\frac{1}{4\left(4 \pi^{2}\right)} d x d\left|k_{\mathrm{T}}\right|^{2} \frac{1}{\sqrt{(1-x)^{2}-\frac{4\left|k_{\mathrm{T}}\right|^{2}}{s}}} \\
& =\frac{1}{4\left(4 \pi^{2}\right)} d x d\left|k_{\mathrm{T}}\right|^{2}\left[\frac{1}{(1-x)_{+}}-\frac{1}{2} \delta(1-x) \ln \frac{4\left|k_{\mathrm{T}}\right|^{2}}{s}\right]+O\left(\left|k_{\mathrm{T}}\right|^{2}\right),
\end{aligned}
$$

where we have introduced the standard plus distribution, implicitly defined by the distributional identity

$$
\int_{0}^{1} d x \frac{1}{(1-x)_{+}} f(x)=\int_{0}^{1} d x \frac{f(x)-f(1)}{1-x} .
$$

Note that because $\left|k_{\mathrm{T}}\right|^{2} \leq s / 4$ the sign of the log in Eq. (35) is such that the contribution proportional to the delta is always positive. If, as mentioned, the squared amplitude behaves as $|M|^{2} \sim \frac{1}{\left|k_{T}\right|^{2}}$, when integrating over the phase space Eq. (35), the $k_{\mathrm{T}}$ integration leads to a double log, which is now clearly seen to arise when both $\left|k_{\mathrm{T}}\right|^{2}$ but also $x \rightarrow 1$, because of the delta.

We now show this explicitly. The amplitude has the form of Eq. 110, but with $k \rightarrow-k$ because the fermions are in the final state, so with the Sudakov parametrization Eq. (25)

$$
|M|^{2}=-\left|M_{0}\right|^{2} e^{2} \frac{8 p_{1} \cdot p_{2}}{\left(p_{1}^{2}-2 k \cdot p_{1}\right)\left(p_{2}^{2}-2 k \cdot p_{2}\right)},
$$

with

$$
\begin{aligned}
& k \cdot p_{1}=\frac{1}{2}[(1-x)-y] p_{1} \cdot p_{2}+\frac{1}{2}[(1-x)+y] p_{1}^{2}=x_{2} p_{1} \cdot p_{2}+x_{1} p_{1}^{2} \\
& k \cdot p_{2}=\frac{1}{2}[(1-x)+y]\left(p_{1} \cdot p_{2}+p_{2}^{2}\right)=x_{1} p_{1} \cdot p_{2}+x_{2} p_{1}^{2} .
\end{aligned}
$$

In the $\left|k_{\mathrm{T}}\right| \rightarrow 0$ limit, using Eq. 32 we get

$$
\begin{aligned}
& x_{1}=\frac{1}{2}[(1-x)+y]=(1-x)+O\left(\left|k_{\mathrm{T}}\right|^{2} / s\right) \\
& x_{2}=\frac{1}{2}[(1-x)-y]=\frac{\left|k_{\mathrm{T}}\right|^{2}}{(1-x) s}\left(1+O\left(\left|k_{\mathrm{T}}\right|^{2} / s\right)\right),
\end{aligned}
$$


where we have assumed for definiteness $y>0$, and the opposite sign would simply amount to interchanging $x_{1}$ and $x_{2}$ (i.e. the collinear ad anticollinear limits). Equations (39) show that $x_{2} \rightarrow 0$ corresponds to the collinear limit, while $x_{1} \rightarrow 0$ to the soft limit, with the two limits interchanged in the anticollinear case in which the negative $y$ solution is chosen.

\subsection{On-shell and off-shell}

For on-shell fermions, $2 p_{1} \cdot p_{2}=s$ and $p_{i}^{2}=0$, so that the matrix element is then given by

$$
\begin{aligned}
|M|^{2} & =-2\left|M_{0}\right|^{2} e^{2} \frac{16}{s\left[(1-x)^{2}-y^{2}\right]} \\
& =-2\left|M_{0}\right|^{2} e^{2} \frac{4}{\left|k_{\mathrm{T}}\right|^{2}},
\end{aligned}
$$

where we have used Eq. (32) and we have provided a factor of 2 in order to account for the two solutions for $y$, which correspond respectively to the collinear or anticollinear regions when $\left|k_{\mathrm{T}}\right| \rightarrow 0$.

Hence, integrating over $x$ with the phase space Eq. (35) we get

$$
\begin{aligned}
\int d \Phi_{k}|M|^{2} & =-\left|M_{0}\right|^{2} \frac{e^{2}}{4\left(4 \pi^{2}\right)} \int d x d\left|k_{\mathrm{T}}\right|^{2}\left[\frac{1}{(1-x)_{+}}-\frac{1}{2} \delta(1-x) \ln \frac{4\left|k_{\mathrm{T}}\right|^{2}}{s}\right] \frac{8}{\left|k_{\mathrm{T}}\right|^{2}} \\
& =-\left|M_{0}\right|^{2} \frac{\alpha}{2 \pi} \ln ^{2} \frac{s}{\mu^{2}},
\end{aligned}
$$

where the first equality holds up to non-logarithmic terms, and the second equality, which holds to double-logarithmic accuracy, is found cutting off the logarithmic integration over $\left|k_{\mathrm{T}}\right|^{2}$ with an infrared regulator (photon mass) $\mu^{2}$; note that the sign follows from the fact that it is the lower limit of integration which provides the $\mu^{2}$ dependence.

We thus get the same double-log result as Eq. (18). The advantage of this choice of frame is that origin of the double log can be traced to the behavior of the phase space Eq. (35) in the simultaneous infrared $x \rightarrow 1$ and collinear $\left|k_{\mathrm{T}}\right| \rightarrow 0$ limit.

Let us now turn to the off-shell case. The denominator of the amplitude is now given by

$$
\begin{aligned}
D & =\left(p_{1}^{2}-2 k \cdot p_{1}\right)\left(p_{2}^{2}-2 k \cdot p_{2}\right) \\
& =s\left[(1-x)^{2}-y^{2}\right]\left[\left(\frac{s}{2}+p_{1}^{2}\right)-\frac{p_{1}^{2}}{x_{2}}\right]\left[\left(\frac{s}{2}+p_{2}^{2}\right)-\frac{p_{2}^{2}}{x_{1}}\right]\left(1+O\left(p_{i}^{2} / s\right)\right) .
\end{aligned}
$$


This immediately implies that when integrating with the phase space Eq. (35) the term proportional to the delta does not contribute: $x_{1}$ vanishes in the $x \rightarrow 1$ limit, so $\lim _{x \rightarrow 1} D=\infty$ because of the second factor in square brackets in Eq. (44). Indeed, there is no longer an infrared singularity when $x \rightarrow 1$, because the off-shellness regulates it.

It is then convenient to write the denominator as

$$
\begin{aligned}
D & =\left(x_{2} \frac{s}{2}-p_{1}^{2}\right)\left(x_{1} \frac{s}{2}-p_{2}^{2}\right)\left(1+O\left(p_{i}^{2} / s\right)\right) \\
& =\frac{1}{1-x}\left[\left|k_{\mathrm{T}}\right|^{2}-p_{1}^{2}(1-x)\right] s\left[(1-x)-\frac{p_{2}^{2}}{s}\right]\left(1+O\left(p_{i}^{2} / s\right)+O\left(\left|k_{\mathrm{T}}\right|^{2} / s\right)\right),
\end{aligned}
$$

where in the second step we have used Eqs. (39-40), in the small $\left|k_{\mathrm{T}}\right|$ limit.

The integrated square amplitude is thus given by

$$
\begin{aligned}
\int d \Phi_{k}|M|^{2} & =-\left|M_{0}\right|^{2} \frac{e^{2}}{4\left(4 \pi^{2}\right)} \int d x d\left|k_{\mathrm{T}}\right|^{2} \frac{1}{(1-x)_{+}} \frac{4 s}{D} \\
& =-2\left|M_{0}\right|^{2} \frac{\alpha}{\pi} \int d x d\left|k_{\mathrm{T}}\right|^{2} \frac{1}{\left[\left|k_{\mathrm{T}}\right|^{2}-p_{1}^{2}(1-x)\right]\left[(1-x)-\frac{p_{2}^{2}}{s}\right]}+\text { non log, }
\end{aligned}
$$

where again we have provided a factor of 2 in order to account for the two (collinear and anticollinear) solutions for $y$. Note that the plus prescription in the first line of Eq. (46) has no effect because the integrand vanishes at $x=1$, as it is clear from Eq. (45).

The integral over $\left|k_{\mathrm{T}}\right|^{2}$ in Eq. (46) is logarithmic about $\left|k_{\mathrm{T}}\right|^{2} \sim p_{1}^{2}(1-x)$, where the first factor in square brackets in the denominator $D$ Eq. (45) vanishes, thus leading to

$$
\int d \Phi_{k}|M|^{2}=-2\left|M_{0}\right|^{2} \frac{\alpha}{\pi} \int d x \ln \left(\frac{s}{2 p_{1}^{2}(1-x)}\right) \frac{1}{(1-x)-\frac{p_{2}^{2}}{s}} .
$$

The integral over $x$ has an infrared singularity regulated by $p_{2}^{2}$ when $(1-x) \sim$ $\frac{p_{2}^{2}}{s}$. The integral over $x$ is thus again double-logarithmic, leading to

$$
\int d \Phi_{k}|M|^{2}=-\left|M_{0}\right|^{2} \frac{\alpha}{\pi} \ln \frac{s}{p_{1}^{2}} \ln \frac{s}{p_{2}^{2}}=-\left|M_{0}\right|^{2} \frac{\alpha}{\pi} \ln \frac{|q|^{2}}{p_{1}^{2}} \ln \frac{|q|^{2}}{p_{2}^{2}}
$$

as in Eq. (24).

It is now clear that the factor two difference between the on-shell result Eq. (42) and the off-shell result Eq. (49) reveals a different underlying physics. 
In the on-shell case, the double log stems from the soft-collinear region, corresponding to the last term in the expression Eq. (35) of the phase space. This is a genuine double log, in that it is due to the square-root factor in the phase space being singular when both the transverse momentum $\left|k_{\mathrm{T}}\right| \rightarrow 0$ and the energy of the emitted photon $E \rightarrow 0$ [i.e. $x \rightarrow 1$, recalling Eq. (29)]. In the off-shell case instead the double log is really coming from the interference of two logarithmic integration regions when the two propagators go on shell, with the phase space now playing no role. These two integration regions correspond to an integral over energy (or $x$ ) Eqs. (33-34) and transverse momentum $\left|k_{\mathrm{T}}\right|$, but they are now decoupled.

\subsection{Exponentiation}

The argument presented in this Section so far concerns only the one-loop or single-emission contributions of Figs. 1,2, so one may wonder whether they apply to all orders, and if so why. Clearly, multiple eikonal emission does exponentiate, as textbook arguments show, but the nontrivial question is what happens to the phase space structure. However, it was recently [1] shown that the phase space for $n$-gluon (and thus also photon) emission in the small $\left|k_{\mathrm{T}}\right|$ has a factorized form which reproduces iteratively the structure Eq. (35).

Specifically, the momenta of the emitted photons can be parametrized as

$$
k_{i}=\alpha_{i} \frac{p_{1}+p_{2}}{2}+y_{i} \frac{p_{1}-p_{2}}{2}+k_{\mathrm{T}}^{i}
$$

so that of course

$$
y_{i}= \pm \sqrt{\alpha_{i}^{2}-\frac{4\left|k_{t}^{i}\right|^{2}}{\hat{s}}} .
$$

Introducing new variables $z_{i}$ through

$$
\alpha_{1}=1-z_{1} ; \quad \alpha_{i}=z_{1} \ldots z_{i-1}\left(1-z_{i}\right), i \geq 2
$$

it can then be shown[1] that

$$
\prod_{i=1}^{n} \frac{d \alpha_{i}}{\sqrt{\alpha_{i}^{2}-4 \frac{\left|k_{T}\right|^{2}}{s}}}=\prod_{i=1}^{n} \frac{d z_{i}}{\sqrt{\left(1-z_{i}\right)^{2}-\frac{4\left|k_{T}\right|^{2}}{z_{1}^{2} \ldots z_{i-1}^{2} s}}}
$$


and that the phase space can be written as

$$
\begin{aligned}
d \Phi_{n+1}\left(p_{1}, p_{2} ; q, k_{1}, \ldots, k_{n}\right) & =\frac{8 \pi^{3}}{\left[4(2 \pi)^{2}\right]^{n+1}} \frac{d q_{t}^{2}}{s} \int d b^{2} J_{0}\left(b\left|q_{t}\right|\right) \\
& J_{0}\left(b\left|k_{\mathrm{T}}^{1}\right|\right) d\left|k_{\mathrm{T}}^{1}\right|^{2} d z_{1}\left[\frac{1}{\left(1-z_{1}\right)_{+}}-\delta\left(1-z_{1}\right) \frac{1}{2} \ln \frac{\left|k_{\mathrm{T}}^{1}\right|^{2}}{s}\right] \ldots \\
& J_{0}\left(b\left|k_{\mathrm{T}}^{n}\right|\right) d\left|k_{\mathrm{T}}^{n}\right|^{2} d z_{n}\left[\frac{1}{\left(1-z_{n}\right)_{+}}-\delta\left(1-z_{n}\right) \frac{1}{2} \ln \frac{\left|k_{\mathrm{T}}^{n}\right|^{2}}{s}\right] \\
& \delta\left(\tau-z_{1} \ldots z_{n}\right)+O\left(\frac{1}{b}\right)
\end{aligned}
$$

where $\tau=\frac{\left|q^{2}\right|}{s}$. The Fourier transform with respect to transverse momentum is necessary in order to factorize the delta function which ensures transverse momentum conservation, but it is clear that the structure of Sect. 4 is then preserved and simply iterated, thereby leading to exponentiation through arguments that are now textbook[15] matter.

Clearly, once the problem is viewed in this way, the exponentiation is seen to have the same origin, both on-shell and off-shell: even though the different origin of the double log is manifested by the factor two difference that we discussed.

As for the RG argument that Wilson asked Roman to construct, it was eventually presented thirty years later[6], as a consequence of the factorization of the integrated amplitude in terms of a factor which contains the soft emissions, and the rest. The underlying physical reason is [7] that in the soft limit the amplitude depends on the variables $\left|k_{\mathrm{T}}\right|^{2}, x$ and $s$ only through the combination $\frac{4\left|k_{\mathrm{T}}\right|^{2}}{s(1-x)^{2}}$, essentially because $\left|k_{\mathrm{T}}^{2}\right|^{\max }=\frac{s(1-x)^{2}}{4}$ is the upper limit of the logarithmic transverse momentum integration. The fact that the square amplitude only depends on one variable then allows for $R G$ improvement with respect to it.

\section{Conclusion}

In summary, Roman's computation amounted to what in modern language would be called the determination of the high-energy behavior of the DrellYan process using reverse unitarity: [16] the inclusive production of a gauge boson in fermion-antifermion annihilation, computed using the cutting rule Eq. (8) to express real radiation phase-space integrals in terms of loops.

What I have done here instead is to compute the real emission contributions directly. With this, I have answered the question that Roman had asked me: the factor two difference between the coefficient of the double 
logarithm in on-shell and off-shell vertex functions (which then to all orders exponentiates) does have a simple physical interpretation. Namely, in the on-shell case the double log is a soft-collinear log, coming from the behavior of phase-space in the region in which the energy and momentum of the emitted photon simultaneously go to zero; while in the off-shell case the double $\log$ is the interference of two independent logarithmic integrations, over the emitted photon energy and transverse momentum, coming from the region where two propagator denominators vanish. It is only in the on-shell case that there exists a genuinely infrared and collinear region.

\section{Added notes}

After publication on the arXiv of the first version of this paper, John Collins pointed out to me that the factor of two disscussed in the present note is also discussed in his QCD treatise [17], and it was surely known in the early days of perturbative QCD: indeed, it is mentioned (in passing) in the introduction of the seminal paper by Mueller [18] in which the Sudakov form factor is computed in QED on-shell, for massive fermions (and photons), beyond the double logarithmic approximation, to all logarithmic orders. This paper was at the origin of subsequent generalizations to QCD by Collins himself [19], which are at the basis of the celebrated Collins-Soper-Sterman early results on QCD factorization [20, 21]. Interestingly, Mueller's paper does altready introduce and exploit renormalization group methods.

The discussion in the QCD book [17] presents a computation of the loop diagram, similar to that performed by Roman [2] and presented in "Landau" [12]; the origin of the factor of two is explained in terms of regions which contribute to the loop integral in the on-shell vs. off-shell case (see in particular Sect. 10.5.3 of the book [17]). In this sense the computation presented here provides a complementary, possibly more "physical" interpretation, to the extent that real emission is physically more intuitive than virtual corrections.

Also, it was recently found that a curious difference of a factor two in Sudakov form factors appears when comparing initial and final state radiation [22].

Interestingly, Mueller [18] does not cite Roman's result. We will leave it to the reader to draw a lesson from this sequence of oblivions and re-discoveries — including my own.

Note added in proof: A few months after submission of this contribution, I was looking into an old review paper that I should know quite well, by my 
late mentor Guido Altarelli [23]. I realized that it contains a section called "The Sudakov form factor of partons", something that I had completely forgotten. I also realized that in this section Eqs. (4) and (5) are both to be found, accompanied by the following sentence: "It is well known that for an off shell quark the exponent differs by a factor of 2". No reference is given, presumably because this fact is so well known.

\section{Acknowledgments}

I am grateful to Giancarlo Ferrera and Paolo Nason for several discussions on the content of this paper, and also for a critical reading of the manuscript. In particular, Giancarlo pointed out to me the discussion of the Sudakov form factor in Landau's treatise [12, while the computation presented in Sect. 2 is due to Paolo, whom I also thank for providing me with detailed notes. I am very grateful to John Collins for correspondence on the subject of this note and for calling my attention to Refs. [18, 19] and especially to the discussion in his book [17]. I also thank Giampiero Passarino for interesting comments and for spotting some typos, Jeffrey Forshaw for pointing out his recent work [22], and Phil Ratcliffe for spotting a typo in an equation and for interesting comments on possible related work by Landshoff and Stirling.

I acknowledge financial support from the European Research Council under the European Union's Horizon 2020 research and innovation Programme (grant agreement n. 740006).

\section{References}

[1] C. Muselli, S. Forte, and G. Ridolfi, Combined threshold and transverse momentum resummation for inclusive observables, JHEP 03 (2017) 106, arXiv:1701.01464.

[2] R. Jackiw, Dynamics at high momentum and the vertex function of spinor electrodynamics, Annals Phys. 48 (1968) 292-321.

[3] V. V. Sudakov, Vertex parts at very high-energies in quantum electrodynamics, Sov. Phys. JETP 3 (1956) 65-71. [Zh. Eksp. Teor. Fiz.30,87(1956)].

[4] B. L. Ioffe, The first dozen years of the history of ITEP Theoretical Physics Laboratory, Eur. Phys. J. H38 (2013) 83-135, arXiv:1208.1386. 
[5] R. Jackiw, Ken Wilson - The Early Years, Int. J. Mod. Phys. A29 (2014) 1430008, arXiv:1312.6634.

[6] H. Contopanagos, E. Laenen, and G. F. Sterman, Sudakov factorization and resummation, Nucl. Phys. B484 (1997) 303-330, hep-ph/9604313.

[7] S. Forte and G. Ridolfi, Renormalization group approach to soft gluon resummation, Nucl. Phys. B650 (2003) 229-270, [hep-ph/0209154].

[8] S. Weinberg, Dynamics at infinite momentum, Phys. Rev. 150 (1966) 1313-1318.

[9] G. F. Sterman, An Introduction to quantum field theory. Cambridge University Press, 1993.

[10] R. Venugopalan, Introduction to light cone field theory and high-energy scattering, Lect. Notes Phys. 516 (1999) 89, nucl-th/9808023. [,89(1998)].

[11] M. Cassandro and M. Cini, Asymptotic limit of vertex functions in perturbation theory, Nuovo Cim. 34 (1964) 1719.

[12] V. B. Berestetskii, E. M. Lifshitz, and L. P. Pitaevskii, QUANTUM ELECTRODYNAMICS, vol. 4 of Course of Theoretical Physics. Pergamon Press, Oxford, 1982.

[13] S. Weinberg, The Quantum theory of fields. Vol. 1: Foundations. Cambridge University Press, 2005.

[14] P. Nason, Sudakov wih photon mass or off-shell fermion cutoff, .

[15] M. E. Peskin and D. V. Schroeder, An Introduction to quantum field theory. Addison-Wesley, Reading, USA, 1995.

[16] C. Anastasiou, K. Melnikov, and F. Petriello, A new method for real radiation at NNLO, Phys. Rev. D69 (2004) 076010, hep-ph/0311311.

[17] J. Collins, Foundations of perturbative QCD, Camb. Monogr. Part. Phys. Nucl. Phys. Cosmol. 32 (2011) 1-624.

[18] A. H. Mueller, On the Asymptotic Behavior of the Sudakov Form-factor, Phys. Rev. D20 (1979) 2037.

[19] J. C. Collins, Algorithm to Compute Corrections to the Sudakov Form-factor, Phys. Rev. D22 (1980) 1478. 
[20] J. C. Collins and D. E. Soper, Back-To-Back Jets in QCD, Nucl. Phys. B193 (1981) 381. [Erratum: Nucl. Phys.B213,545(1983)].

[21] J. C. Collins, D. E. Soper, and G. F. Sterman, Does the Drell-Yan Cross-section Factorize?, Phys. Lett. 109B (1982) 388-392.

[22] J. R. Forshaw, J. Holguin, and S. Plätzer, Parton branching at amplitude level, arXiv:1905.08686. [JHEP08,145(2019)].

[23] G. Altarelli, Partons in Quantum Chromodynamics, Phys. Rept. 81 (1982) 1. 